Essomba, M.A. (2014). Políticas de escolarización del alumnado de origen extranjero en el estado español hoy. análisis y propuestas. Revista Electrónica Interuniversitaria de Formación del Profesorado, $17(2), 13-27$.

DOI: http://dx.doi.org/10.6018/reifop.17.2.198771

\title{
Políticas de escolarización del alumnado de origen extranjero en el estado español hoy. análisis y propuestas
}

\author{
Miquel Àngel Essomba
}

Coordinador general de la red SIRIUS sobre políticas de inmigración y educación de la UE

\section{Resumen}

Este texto refleja los puntos fuertes del debate de la red SIRIUS en el estado español en 2013. El objetivo de dicho encuentro fue diagnosticar el estado actual de implementación de las políticas de escolarización básica obligatoria del alumnado de origen extranjero, y explorar soluciones, aportar experiencias y buenas prácticas que puedan servir de paliativo a las problemáticas identificadas. El documento sigue una estructura lógica vertebrada en dos partes. La primera está dedicada a recoger las voces en diálogo de los participantes en el encuentro, atendiendo un esquema que sigue una pauta relacionada con el mismo proceso de escolarización de estos alumnos: políticas relativas al acceso al sistema, a su permanencia y al proceso de integración social. La segunda parte está dedicada a plantear un modelo de innovación en la política educativa dirigida al alumnado de origen extranjero, que parte del análisis efectuado por los integrantes en la reunión, pero que también suma reflexiones y aportes teóricos construidos en el marco más internacional de la red SIRIUS, lo que permite un encaje local-global óptimo a la hora de diseñar propuestas de futuro. Al final se ofrecen algunas pistas de futuro sobre posibles caminos de investigación y de acción relacionados con las políticas educativas de atención al alumnado de origen extranjero.

\section{Palabras clave}

Política educativa; alumnado de origen extranjero; sistema educativo; integración social.

Contacto

Miquel A. Essomba, miquelangel.essomba@uab.cat, Facultad de Ciencias de la Educación.Edificio G-6. Campus de Bellaterra - UAB- 08193 Bellaterra (Cerdanyola del Vallès).

El proyecto SIRIUS se enmarca dentro del programa Lifelong Learning de la Comisión Europea. El código de convocatoria competitiva es "Call for proposals EAC/01/2011 - Decision no 1357/2008/EC of the European Parliament and of the Council”. Para más información, véase existir. véase www.sirius-migrationeducation.org 


\title{
Educational policies of attention to students with a migrant background in Spanish. Analysis and proposals
}

\begin{abstract}
This text reflects key issues discussed in the Spanish SIRIUS meeting in 2013. The aim of the meeting was to assess the current state of implementation of the policies of compulsory basic schooling for students with a migrant background, and explore solutions to provide experiences and best practices that can serve as a palliative to the identified problems. The document follows a logical structure divided in two parts. The first one is devoted to collect the participants' voices on the process of education of these students: policies on access to the system, its permanence and the social process of integration. The second part is devoted to present a model of innovation in education policy addressed to students with a migrant background. It also adds reflections and theoretical contributions built on the framework of the international SIRIUS network, allowing a local-global optimal fit for the future. Finally some hints are expressed on future research projects and actions related to the educational policies of attention to students with a migrant background.
\end{abstract}

\section{Key words}

Educational policy; students migrant background; educational system; social integration.

\section{Introducción}

El 4 de octubre de 2013 tuvo lugar en Madrid la reunión estatal de la red SIRIUS, organización europea dedicada a analizar y mejorar la implementación de políticas de inmigración y educación de la UE. El objetivo de dicho encuentro fue diagnosticar el estado actual de implementación de las políticas de escolarización básica obligatoria del alumnado de origen extranjero, y explorar soluciones, aportar experiencias y buenas prácticas que puedan servir de paliativo a las problemáticas identificadas.

El debate fue rico e intenso, tanto en contenidos como en formato, y fue llevado a cabo por una treintena de agentes sociales y profesionales diversos: académicos, responsables de administraciones públicas y de entidades sociales, procedentes todos de tres comunidades autónomas caracterizadas por su diversidad en la aplicación de políticas públicas y por concentrar la gran mayoría del alumnado de origen extranjero de todo el estado (Cataluña, Andalucía y Madrid).

En este texto deseamos reproducir, de manera sistematizada, los principales temas tratados y las respuestas apuntadas, de modo que dicho documento pueda resultar un diagnóstico aproximado al estado actual de implementación de las políticas educativas relativas a la escolarización básica obligatoria del alumnado de origen extranjero en el estado español.

El documento sigue una estructura lógica vertebrada en dos partes. La primera está dedicada a recoger las voces en diálogo de los participantes en la reunión, atendiendo un esquema que sigue una pauta relacionada con el mismo proceso de escolarización de estos alumnos: políticas relativas al acceso al sistema, a su permanencia y al proceso de integración social. 
La segunda parte está dedicada a plantear un modelo de innovación en la política educativa dirigida al alumnado de origen extranjero, que parte del análisis efectuado por los integrantes en la reunión, pero que también suma reflexiones y aportes teóricos construidos en el marco más internacional de la red SIRIUS, lo que permite un encaje localglobal óptimo a la hora de diseñar propuestas de futuro.

Antes de entrar en materia, cabe decir que el análisis de políticas y el planteamiento de propuestas se centra en la escolarización básica obligatoria (6-16 años) de este alumnado de origen extranjero, pero ello no significa que deba abandonarse el estudio de políticas dirigidas a otras etapas escolares, formativas y educativas (educación infantil, formación profesional, estudios universitarios, formación artística, educación de personas adultas, educación en el tiempo libre). Dicho estudio queda pendiente para ocasiones posteriores.

\section{Primera parte. análisis de las políticas educativas en materia de acceso, permanencia e integración social del alumnado de origen extranjero en el estado español}

\section{Políticas de acceso a la escolarización}

Desde un punto de vista normativo, el derecho de los hijos e hijas de extranjeros a la escolarización básica obligatoria en el estado español siempre se ha regido por el principio de igualdad y la no vulneración del derecho universal de estos menores a la educación (artículo 26.1 de la declaración universal de los derechos humanos).

En este sentido, cualquier menor extranjero entre los 6 y los 16 años debe acogerse a lo estipulado en materia de educación que sus iguales de nacionalidad española. Así queda reflejado en la última reforma de la ley de extranjería, la ley orgánica 2/2009, en la cual se modifica el artículo 9.1 de la anterior y declara que

"los extranjeros menores de dieciséis años tienen el derecho y el deber a la educación, que incluye el acceso a una enseñanza básica, gratuita y obligatoria (...) Este derecho incluye la obtención de la titulación académica correspondiente y el acceso al sistema público de becas y ayudas en las mismas condiciones que los españoles".

A su vez, vincula dicho derecho y deber a la escolarización con la obtención de la documentación legal necesaria para residir en territorio español por parte de sus progenitores:

"los extranjeros residentes que tengan en España menores a su cargo en edad de escolarización obligatoria, deberán acreditar dicha escolarización, mediante informe emitido por las autoridades autonómicas competentes, en las solicitudes de renovación de su autorización o en su solicitud de residencia de larga duración" (artículo 9.4).

Por este motivo, debido a la equiparación legal entre menores extranjeros y españoles con respecto al derecho a la educación, el acceso también se ha regido por el mismo principio de igualdad. Así queda reflejado en el articulado no modificado de la ley orgánica 3/2006 de educación, en cuyo artículo 78.1 establece que

"corresponde a las Administraciones públicas favorecer la incorporación al sistema educativo de los alumnos que, por proceder de otros países o por cualquier otro motivo, se incorporen de forma tardía al sistema educativo español. Dicha incorporación se garantizará, en todo caso, en la edad de escolarización obligatoria" y en el 84.3 se determina que "en ningún caso habrá discriminación por razón de 
nacimiento, raza, sexo, religión, opinión o cualquier otra condición o circunstancia personal o social".

Sin embargo, los participantes en la reunión de SIRIUS manifestaron que tal principio se ve reiteradamente vulnerado en la realidad cotidiana, y la dinámica de la sociedad española desarrolla problemáticas asociadas al acceso a la escolarización de este alumnado. La consecuencia inmediata es el incremento potencial del riesgo a la hora de que el sistema educativo cumpla su función de proporcionar oportunidades para la igualdad. Se constata, en definitiva, un hiato entre norma y sociedad, y ello pone en tela de juicio la posibilidad de que el sistema educativo actúe como constructor de cohesión social.

De acuerdo con las voces en diálogo, esta falta de correspondencia entre norma y sociedad puede verse reflejada en dos dinámicas sociales contrarias a la cohesión:

- Dualización y guetización del sistema: Se afirma que se mantiene el desequilibrio de ratios en la escolarización del alumnado de origen extranjero según titularidad del centro. Los datos ministeriales corroboran esta percepción. Según fuentes del MECD, si bien en 2012 la distribución global del alumnado de régimen general según titularidad se distribuía con un $68.3 \%$ para los centros públicos, un $25,4 \%$ los concertados y un $6.3 \%$ los privados, los centros públicos escolarizaban para las enseñanzas de régimen general al $82.5 \%$ del alumnado extranjero, mientras que los concertados ese porcentaje se limitaba al $13.7 \%$ y el de los privados al $3.8 \%$ (MECD, 2013)

- Desarticulación: Se afirma que se mantienen problemas en el acceso a estudios postobligatorios por parte del alumnado de origen extranjero. Existe la percepción de elevado desequilibrio en la proporción de alumnado de origen extranjero y español que accede a la enseñanza secundaria postobligatoria. A falta de estudios más concluyentes de ámbito estatal, un estudio de ámbito autonómico refuerza esta percepción, y pone de manifiesto que la "población extranjera es la que aparece como más infrarrepresentada, especialmente en el bachillerato, y con niveles más altos de abandono escolar prematuro" (Sánchez Martí y Sandín, 2013)

En cualquier caso, se señala la necesidad de atender a las políticas de acceso a la escolarización del alumnado de origen extranjero como una prioridad, no sólo en calidad de su dimensión administrativa sino también de su fuerte carga política y social.

Con respecto a su carga política, las voces en diálogo subrayan la importancia de establecer políticas de reequilibrio. Especialmente las voces representantes de la administración local reivindicaron el papel de las que fueron comisiones de escolarización, responsables de velar por un acceso a la escolarización basado en la igualdad de oportunidades, con independencia del origen del alumnado, y garantizando procesos de equidad a la hora de distribuirlo.

Con relación a su carga social, se hace hincapié en las políticas de acogida, que van más allá de dispositivos individuales o institucionales, y abarcan un espectro más amplio que cubre el conjunto del territorio donde el recién llegado se ubica. Un ejemplo de buena práctica lo constituye el programa de planes educativos de entorno de la Generalitat de Catalunya, el cual cubre un complejo entramado de complicidades institucionales entre poderes públicos locales, entidades sociales y cívicas, centros escolares y administración educativa de un mismo territorio, con el fin de que el acceso escolar sea también una oportunidad para el acceso social.

La reflexión sobre el acceso a la escolarización del alumnado de origen extranjero concluyó con la identificación de tres importantes retos de avance: 
- Si bien se asume por lo general la necesidad de trabajar en red, se echa en falta la existencia de modelos operativos concretos que permitan visualizar cómo llevar a cabo un trabajo en red de forma óptima. Se sugiere identificar distintos modelos de trabajo en red a nivel europeo para observar cómo trasladar buenas prácticas a municipios españoles. Se señala el informe NESET "Alliances for inclusión: cross-sector policy synergies and inter-professional collaboration in and around schools" (Edwards y Downes, 2013) como un buen punto de partida.

- La falta creciente de recursos para la educación plantea la duda respecto a la conveniencia de avanzar en la dimensión política y social del acceso del alumnado de origen extranjero a la escuela. La continuidad de las comisiones de escolarización, o de iniciativas como los planes educativos de entorno, dependen del mínimo de recursos exigible. La sostenibilidad no siempre es posible debido a esta falta.

- Las administraciones educativas manifiestan dificultades a la hora de realizar un seguimiento sistematizado de las políticas de matriculación, de acogida y de transición entre etapas educativas. En este sentido, se hace necesario vertebrar un sistema que facilite dichos datos de libre acceso a los investigadores y los agentes sociales, con el fin de utilizarlos con fines de mejora.

\section{Políticas de permanencia en el sistema educativo}

La permanencia del alumnado de origen extranjero en el sistema educativo se vincula a la capacidad de dicho sistema de proporcionar una escolarización de calidad. La búsqueda de esta calidad se refleja en el ordenamiento jurídico español desde hace aproximadamente una década. Ya la ley orgánica 3/2006 de educación lo recoge en su artículo 78.2:

"las Administraciones educativas garantizarán que la escolarización del alumnado que acceda de forma tardía al sistema educativo español se realice atendiendo a sus circunstancias, conocimientos, edad e historial académico, de modo que se pueda incorporar al curso más adecuado a sus características y conocimientos previos, con los apoyos oportunos, y de esta forma continuar con aprovechamiento su educación".

Los principios rectores de dicha calidad responden a una estrategia de atención a la diversidad basada en tres grandes aspectos: la atención individualizada, la inclusión y la colaboración familia-escuela (artículo 79 de la misma ley).

Sin embargo, también en este caso las voces en diálogo de la reunión señalan la distancia existente entre la norma y la realidad de los centros escolares. Es decir, se manifiesta una preocupación por los peores resultados académicos del alumnado de origen extranjero respecto al de origen español, así como su proporcionalmente elevada tasa de absentismo y abandono escolar prematuro. Las investigaciones de ámbito internacional abonan estas tesis desde hace tiempo (OCDE, 2006; EURYDICE, 2009; OCDE, 2012). No obstante, en nuestro contexto existen estudios longitudinales recientes que ponen de manifiesto que, si bien se ratifica el inferior rendimiento académico del alumnado de origen extranjero, se cuestiona que el abandono escolar prematuro sea superior al de los de origen español (Portes y Aparicio, 2013).

En cualquier caso, para garantizar una calidad que blinde la permanencia en el sistema de este alumnado, las voces en diálogo apuntan a la necesidad de avanzar en dos tipos de políticas: de prevención del fracaso escolar y de acompañamiento en los procesos de transición.

Con respecto a las políticas dirigidas a la prevención del fracaso escolar del alumnado de origen extranjero, se considera básico desplegar políticas de gobernanza del sistema 
favorables al desarrollo de condiciones diversificadas para el éxito. Los proyectos de aprendizaje-servicio o las comunidades de aprendizaje aparecen como propuestas de diversificación curricular óptimas para fomentar el éxito escolar de este alumnado en particular, y de todos los alumnos en general. A su vez, también se subraya la importancia de las actividades de orientación y de acción tutorial como espacios privilegiados para trabajar conjuntamente entre alumnado, familia y profesorado para el éxito.

Con relación al acompañamiento en los procesos de transición, se identifican dos momentos clave en los cuales se observan dificultades por parte del alumnado de origen extranjero para completar dicha transición en condiciones de igualdad respecto a sus iguales de origen español. Hablamos de los procesos de transición en el marco de la enseñanza básica obligatoria (de primaria a ESO) y en el seno de la enseñanza secundaria (de obligatoria a postobligatoria). Políticas conducentes a paliar tales fenómenos serían necesarias en todos los territorios.

Para que las políticas de prevención y de acompañamiento dispongan no sólo de oportunidades para su despliegue, sino también para su sostenibilidad en el tiempo, se indican cuatro condiciones estructurales que deben ser consideradas: autonomía de gestión curricular, coordinación interdepartamental, aprendizaje para la participación de los adultos desde la libertad y diversificación de los equipos profesionales.

La reflexión sobre la calidad de la escolarización de estos alumnos, que refuerce su permanencia en el sistema, concluye con la identificación de tres importantes retos de avance:

- El éxito escolar del alumnado extranjero, vinculado a su permanencia en el sistema hasta completar como mínimo un ciclo de estudios postobligatorios, no sólo tiene que ver con el tiempo escolar sino también con el extraescolar. La posibilidad de contar con recursos culturales y sociales que complementan y acompañan el proceso de escolarización es vital para este tipo de alumnado a la hora de prevenir su fracaso, debido sobre todo a sus niveles de capital económico, social y cultural con relación a sus iguales de origen español.

- Resulta necesario definir un modelo social hegemónico que entienda que la presencia de alumnado de origen extranjero en las aulas no representa necesariamente una dificultad para el proceso de escolarización del conjunto del alumnado. Los procesos de orientación y acompañamiento no sólo se deben focalizar en los de origen extranjero sino sobre el conjunto.

- Asimismo, y siguiendo con este argumento, se destaca la necesidad de frenar la reducción del gasto en educación, puesto que si bien el gasto no es suficiente para apuntalar el éxito escolar de un sistema, sí es necesario (Calero, 2008).

\section{Políticas para el fomento de la integración social desde la escolarización}

No debemos perder de vista que las políticas educativas que velan por el derecho a la educación del alumnado de origen extranjero, así como por su acceso y permanencia en condiciones de igualdad de oportunidades, lo que buscan en esencia es el objetivo de la integración social de éstos: "los poderes públicos promoverán que los extranjeros puedan recibir enseñanzas para su mejor integración social” (artículo 9.3 de la ley orgánica 2/2009 de reforma de la Ley Orgánica 4/2000 sobre derechos y libertades de los extranjeros en España y su integración social). 
Por ello, la mirada de las voces en diálogo durante la reunión no solamente se dedicó a realizar un análisis desde dentro del sistema sino que también se dirigió a observar otras dimensiones que de forma tangencial al proceso educativo van creando condiciones para posibilitar que el éxito educativo de estos alumnos se traduzca en su integración social efectiva. En este sentido, dos dimensiones políticas aparecen como las más relevantes: los recursos utilizados, y la construcción de convivencia.

Las políticas de gestión de recursos aparecen como recurrentes y relevantes a lo largo de toda la discusión y, como ya señalamos en el apartado anterior, estas políticas son claves para el éxito en la escolarización de este alumnado. Para apuntalar un éxito escolar conducente a la integración social, las voces en diálogo proponen reforzar tres tipos de políticas dirigidas a la gestión de recursos:

- Políticas de incremento de recursos indirectos, es decir, incremento de más personal que pueda llevar a cabo los programas de atención individualizada, de diversificación curricular o de acompañamiento en los procesos de transición. Puesto que dichos programas no contienen sólo una carga escolar, sino también social, la presencia de más personal que los atienda facilita la integración.

- Políticas de incremento de recursos directos, es decir, incremento de becas y ayudas. El alumnado de origen extranjero, de acuerdo a la ley (ley orgánica 2/2009 de reforma de la Ley Orgánica 4/2000 sobre derechos y libertades de los extranjeros en España y su integración social), debe estar en condiciones de igualdad con respecto a la obtención de este tipo de recursos: el derecho a la educación de los extranjeros menores de dieciséis años "incluye la obtención de la titulación académica correspondiente y el acceso al sistema público de becas y ayudas en las mismas condiciones que los españoles" (artículo 9.1).

- Políticas de incremento de capital cultural del sistema, lo que implica entre otras cosas prestar una atención preferente a las políticas de formación del profesorado. Dicha formación no debe concentrarse en aspectos estrictamente tecnológicos de la profesión sino también éticos - resulta imprescindible abordar al profesorado en su dimensión de ciudadano con respecto a sus estereotipos y prejuicios, y su actitud a la hora de generar inconscientemente mecanismos de preferencia endogrupal que excluyen al alumnado extranjero de las dinámicas generadas y construyen un universo simbólico de exclusión implícita dentro de las aulas donde el alumnado de origen extranjero tiene presencia.

Las políticas promotoras de convivencia son otro de los aspectos fundamentales que, de forma indirecta, contribuyen al éxito educativo del alumnado de origen extranjero. En la medida que se fomentan políticas dirigidas a fomentar la convivencia más allá de la coexistencia (Giménez, 2010), se incrementan las oportunidades para que el impacto de la escolarización sobre la integración social surja efecto. En este sentido, las voces en diálogo señalan la necesidad de atender a tres tipos de políticas que permitan avanzar en ese terreno:

- Políticas dirigidas a combatir la invisibilización y, por ende, la desatención de determinados perfiles de alumnado de origen extranjero. Nos referimos por ejemplo a aquellos que, por el motivo de ser nietos o bisnietos de primeros migrantes, quedan invisibilizados en su proceso complejo de construir identificaciones y relaciones sociales sin tener en cuenta su bagaje cultural familiar. También se hace mención de aquellos alumnos de origen extranjero que, por encontrarse en territorios con baja presencia de población extranjera, no se llevan a cabo acciones para atenderles y se consuman procesos de asimilación encubierta. 
- Políticas dirigidas a vertebrar un modelo de gobernanza escolar que resulte favorable a la construcción de convivencia. Por este motivo, resulta positivo resaltar el valor básico de la comunidad educativa como un espacio natural de integración social más allá de los aprendizajes escolares, y apostar por un modelo de liderazgo distribuido, que permita oportunidades a todos de experimentar el reto de participar. También se subraya la importancia de promover políticas dirigidas a la formación de los miembros de la comunidad educativa en habilidades sociales relacionadas con la mediación.

- Políticas dirigidas a priorizar la satisfacción de necesidades de integración social del alumnado de origen extranjero en edad adolescente. Las voces en diálogo apuntan que se trata de un momento evolutivo clave de las personas en el cual se sientan las bases de una construcción armónica de la identidad cultural (Torrabadella y Tejero, 2005), y pueden definirse expectativas futuras de integración o de exclusión.

\section{Hacia un modelo integral de políticas educativas de atención al alumnado de origen extranjero}

\section{La innovación política como clave para el éxito}

La educación del alumnado de origen extranjero, como vemos, aparece como un reto básico para el desarrollo social desde el punto de vista de todos los participantes en la reunión. Sin embargo, las condiciones globales y complejas del mundo actual, así como el contexto del estado español en estos momentos, exigen la implementación de procesos de innovación política. No se pueden diseñar y aplicar políticas para una nueva situación social desde antiguos planteamientos.

La posibilidad de generar oportunidades para la igualdad, no sólo en el plano normativo que ya hemos visto que existe - sino en la praxis social requiere de nuevas reglas. El reto es enorme, puesto que los sistemas educativos por lo general tienden ellos mismos a ser fuente de desigualdad:

"como (...) las investigaciones sugieren, las identidades étnicas y la situación económica están fuertemente correlacionados y los estudiantes blancos y de clase media están siendo favorecidos por las nuevas políticas de educación con efectos significativos en la educación que se pone a disposición (...). Las políticas que han tratado de compensar estos efectos negativos sólo han tenido un éxito marginal" (Beach y Sernhede, 2011).

Por ello, las voces en diálogo asumen una de las tesis fundamentales que subyacen al discurso que SIRIUS ha elaborado al entorno de las políticas educativas dirigidas al alumnado de origen extranjero: la clave no está en el "qué" sino en el "cómo" se debe transitar del diseño a la implementación de las políticas.

No podemos esperar que el sistema educativo transforme a la sociedad - en realidad, es la sociedad la que transforma al sistema educativo. Por ello, la innovación política que se requiere para apuntalar el éxito escolar del alumnado de origen extranjero debe surgir a partir de tres grandes principios:

- Promover un enfoque comunitario en lugar de un enfoque escolar, mediante el reconocimiento de la importancia de todos los actores de la comunidad en la educación.

- Integrar a todos estos actores en una red articulada de profesionales en la cual lo pertinente no es "quién hace qué", sino "cómo lo hacemos juntos". 
- Crear nuevos formatos de escolarización bajo el principio de inclusión.

De las políticas educativas basadas en la escuela a las políticas educativas basadas en la comunidad

Por lo general, las voces en diálogo constatan que las políticas educativas siguen siendo diseñadas a partir de un enfoque de la escuela tradicional. No se acaba de romper del todo la identificación entre educación y escolarización, y los recursos educativos de los miembros de la comunidad siguen sin disfrutar de un estatus privilegiado a los ojos de la sociedad. Esta tendencia, obviamente, ha sido trasladada a las políticas dirigidas al alumnado de origen extranjero. La mayoría de ellas se concentran en la escuela en sí misma. Si bien la evidencia empírica los ha revelado como necesarios, también se constata que no resultan suficientes. El motivo es que un focalización exclusiva en la escuela limita las posibilidades de acercarse a las causas subyacentes que existen, pongamos por ejemplo, detrás del abandono escolar prematuro. Coincidimos con Lyche (2010) cuando afirma que

"las medidas preventivas, por tanto, deben tener en cuenta no sólo la causa visible directa de deserción escolar, sino también las causas subyacentes que influyen en el proceso acumulativo de desvinculación del estudiante que en última instancia conduce a la decisión de abandonar la educación o la formación".

En consecuencia, la innovación política en materia de escolarización del alumnado extranjero tiene más que ver con la articulación entre las políticas que afectan a la escuela y su comunidad de referencia, que con el diseño e implementación de una nueva batería de políticas dirigidas a la comunidad como complemento. Ello requiere de un liderazgo sólido y capaz de llevar a cabo dicha articulación mediante lo que podemos denominar un proceso de contextualización:

"contextualización significa todo sobre las tácticas que los líderes del sistema utilizan para la adaptación de las intervenciones necesarias a su contexto específico. Nuestra investigación muestra que el objetivo primordial de los líderes del sistema en la contextualización de las intervenciones es por lo general para obtener el apoyo necesario de los distintos grupos de interés para las intervenciones que se están realizando" (Mourshed, Chijioke y Barber, 2011).

Las voces en diálogo ponen de manifiesto que los espacios promovidos por la red SIRIUS pueden resultar significativos para experimentar buenas prácticas de innovación política en este sentido, siempre con relación al acceso, la permanencia y la integración social del alumnado de origen extranjero como protagonista de su propio proceso educativo. En este sentido, y a modo de resumen, se propone que la innovación en la materia se lleve a cabo mediante políticas destinadas a:

- coordinar un sistema escolar articulado dentro de una comunidad en lugar de políticas dirigidas a las escuelas de forma segregada respecto al resto.

- facilitar la participación estratégica de las familias del alumnado de origen extranjero, mediante la creación de un sentido de pertenencia, y el establecimiento de una relación positiva.

- reforzar el papel educativo de los grupos y las instituciones de la comunidad de referencia de las escuelas, poniendo de relieve su papel educativo. 
De las políticas educativas de naturaleza sectorial a las políticas educativas de naturaleza sistémica

La racionalidad analítica intrínseca al modelo científico occidental impregna a las ciencias sociales y a sus prácticas derivadas de linealidad, fragmentación y sectorización. Las políticas educativas no se escapan a dicha influencia, es más, las prácticas que de ellas se derivan no se entienden de otra manera que no sea mediante la aplicación de reglas organizativas propias del taylorismo.

De acuerdo con estos planteamientos de la teoría clásica de las organizaciones, la mayoría de los sistemas educativos se han estructurado de acuerdo con la regla del "uno": un grupo-clase, un maestro, una asignatura, una lección, un libro, un aula. Esta cultura organizativa, que plasma la implementación de las políticas educativas en la realidad cotidiana, aparece a las voces en diálogo como disfuncional a la hora de proporcionar una calidad adecuada en el proceso de escolarización del alumnado de origen extranjero.

Por ello la innovación pasa porque quienes diseñan e implementan políticas superen una perspectiva estructural-funcional del sistema educativo, y se imbuyan de una perspectiva más relacional, en la cual la clave del éxito no reside en los componentes del sistema sino en el sistema de relaciones que éstos establecen entre sí, dentro y fuera del contexto escolar:

"muchos de los sistemas de nuestra muestra han creado una pedagogía en la que los maestros y los líderes de la escuela trabajan juntos para integrar las rutinas que nutren la excelencia educacional y el liderazgo. Ellos integrar rutinas de instrucción y la excelencia del liderazgo en la comunidad educativa, haciendo pública la práctica del aula, y los profesores se convierten en los formadores de sus compañeros" (op.cit.).

Lo comentado sirve para el conjunto del alumnado, pero no hace falta decir que tal planteamiento de la educación es aún más disfuncional para el alumnado de origen extranjero. A este alumnado, además de aplicársele la regla del "uno", además se le añade la regla del "especial" (un alumno especial, que debe seguir un programa especial, en un aula especial, con un profesor especial), lo que acaba dificultando en muchas ocasiones la permanencia y sobre todo la deseada integración social de éste.

No obstante, y siendo justos, las voces en diálogo de la reunión no consideran que esta política de atención individualizada sea negativa a priori. Tan o más importante que el enfoque individualizado es observar, como venimos comentando, el sistema de relaciones dentro del cual se contextualiza dicha atención individualizada. Emerge entonces una perspectiva que fomenta la innovación en este aspecto y que podemos cualificar como sistémica:

"sistémico no significa que los diversos elementos se puedan describir como vinculados sin más. Eso sólo es sistémico en teoría. Es la práctica lo que cuenta. Por lo tanto, las estrategias sistémicas requieren apoyo y esfuerzos de mejora en el ámbito de todas las escuelas y todos los distritos. ( ... ) La capacitación profesional, el trabajo en grupo y una pedagogía profunda, acelerada por la tecnología, son en efecto procesos que sustentan, de hecho reclaman, a todas las escuelas que participen en la mejora de su práctica" (Fullan, 2011).

La red SIRIUS, tanto desde una perspectiva estatal como también europea, se revela como un espacio óptimo para ir infundiendo el espíritu y la práctica de una política educativa pensada y aplicada en clave sistémica para la mejora de la escolarización del alumnado de origen extranjero. De hecho, la propia estructura original de esta red ya representa en sí misma esta perspectiva, puesto que como ya hemos explicado al principio ésta está compuesta por responsables políticos, investigadores y miembros de la sociedad civil a la 
vez. Las voces en diálogo apuntan, en este sentido, que la innovación promotora del paso de una naturaleza sectorial del sistema educativo a otra sistémica pasa por unas políticas destinadas a:

- Formar al profesorado en pensamiento estratégico - no sólo aprender a enseñar al alumnado de origen extranjero, sino también aprender a hacer que su proceso de aprendizaje resulte significativo para éstos.

- Facilitar el desarrollo de las escuelas como comunidades profesionales de aprendizaje (Hargreaves, 2003), donde el profesorado puede disfrutar de las condiciones que permiten aprender unos de otros.

- Regular medidas de lucha contra la discriminación en la escuela desde un enfoque sistémico - etnia, clase y género como epifenómenos de un factor causante común vinculado con la discriminación y la exclusión.

De las políticas educativas promotoras de integración a las políticas educativas promotoras de inclusión

Ya han pasado dos décadas desde que los sistemas educativos en general, y el español en particular, han invertido esfuerzos en dejar atrás el modelo de atención a la diversidad de la integración educativa para asumir progresivamente el de inclusión educativa. La inclusión educativa del alumnado de origen extranjero se juega en todos los terrenos: el acceso, la permanencia y por supuesto la integración social.

Precisamente este alumnado se ve favorecido por políticas que promuevan la educación inclusiva, ya que esta educación, más allá de la escolarización, provee una necesaria normalización que evite la estigmatización innecesaria:

"la educación inclusiva es un proceso continuo cuyo objetivo es ofrecer una educación de calidad para todos, respetando la diversidad y las diferentes necesidades y capacidades, características y expectativas de aprendizaje de los estudiantes y sus comunidades, así como la eliminación de todas las formas de discriminación" (UNESCO, 2008).

Pero la educación inclusiva resulta beneficiosa para el alumnado de origen extranjero porque aporta un efecto positivo de socialización en vez de una individualización salvaje:

"la educación se ha convertido cada vez más en individualizada para permitir cierta movilidad ascendente a los alumnos que lo desean y que tienen acceso al capital cultural y social que lo permite (...) hay un problema en el nuevo milenio donde las escuelas se han convertido en incubadoras de individualismo competitivo y de movilidad individual" (Beach y Dovemark, 2009).

Frente a la perspectiva cada vez más individualista en clave de competitividad, de la cual el alumnado de origen extranjero corre el riesgo de descolgarse, las voces en diálogo manifiestan la necesidad de forjar una innovación política que focalice la socialización como misión fundamental del sistema educativo para la integración social de estos alumnos.

Es necesario que la educación inclusiva sea vista como un espacio donde las personas pueden satisfacer sus necesidades, mediante patrones interactivos de aprendizaje y participación en actividades de cooperación en la construcción del conocimiento. Las voces en diálogo coinciden en señalar que una política de educación inclusiva promueve que el aprendizaje del alumnado sea considerado por encima de la enseñanza del profesorado, y permita a los alumnos de origen extranjero disponer de mayores oportunidades para ser vistos desde sus potencialidades y no de sus carencias coyunturales. La red SIRIUS puede 
facilitar alternativas de innovación política para que ello sea una realidad, mediante la propuesta de políticas destinadas a:

- Evitar los itinerarios académicos diferenciados prematuros, que vulneran el principio de comprensividad propio de un sistema basado en una educación inclusiva.

- Promover un enfoque de derechos humanos y de educación para la ciudadanía como marco óptimo de reglas del juego de la convivencia presente y futura.

\section{El futuro del análisis de políticas educativas de atención al alumnado de origen extranjero}

Este texto ha pretendido ofrecer, de manera sistematizada y ampliada, las conclusiones principales de la red SIRIUS en su encuentro estatal de 2013. Ya sea teniendo al alumnado de origen extranjero como foco de atención (primera parte), ya sea poniendo énfasis en la innovación política como eje de análisis y propuesta (segunda parte), el doble enfoque retrospectivo y prospectivo desarrollado permite disponer de una mirada actualizada sobre el tema.

Como ya apuntamos también al principio, muchos son los temas que quedan en el tintero pendientes de análisis en clave de políticas educativas, y sobre los ya expuestos muchas son las evidencias empíricas que se requieren para validar lo que en una reunión fueron más bien percepciones originadas desde el contexto propio y la subjetividad no expuesta a desarrollos y profundizaciones posteriores.

Nos queda claro, a pesar de todo, que desde una perspectiva de investigación se necesita avanzar más y mejor en como mínimo los siguientes terrenos:

1. Un estudio profundizado sobre el nuevo papel de los poderes públicos locales y del territorio en el contexto actual de recortes de competencias en materia de educación a los municipios. Sin duda alguna, un obstáculo a todo lo descrito a la hora de garantizar el ejercicio efectivo del derecho a la educación del alumnado de origen extranjero.

2. Información sistematizada que permita la monitorización, el seguimiento, el control y la evaluación y, en su caso, la denuncia de los procesos de implementación y de innovación política en materia de educación y extranjería. Si no se sabe lo que pasa, no puede analizarse, criticarse, ni proponer mejoras.

3. Creación de experiencias de terreno basadas en sistemas de relaciones significativos, cercanos, de clara referencia para los alumnos de origen extranjero, y que permitan incrementar su autonomía de forma progresiva. Ir a la búsqueda de su capital social y cultural oculto, invisibilizado, en tiempos de profundo revés económico en todas las esferas en general, y en la de los ciudadanos de origen extranjero en particular.

A todo ello se dedicará la red SIRIUS, en el estado español y en la UE, en el presente y en el futuro.

\section{Bibliografía}

Beach, D. \& Dovemark, M. (2009). Making right choices: An ethnographic investigation on creativity and performativity in four Swedish schools. Oxford Review of Education, $35: 689-704$. 
Beach, D. \& Sernhede, O. (2011). From Learning to Labour to Learning for Marginality: School Segregation and Marginalisation in Swedish Suburbs. British Journal of Sociology of Education, 32, $2: 257-274$.

Calero, J.(2008).¿Es realmente irrelevante el gasto educativo? Diario El País; 31 de marzo de 2008.

Council conclusions of 26 and 27 November 2009 on the education of children with a migrant background.

Edwards, A. y Downes, P. (2013). Alliances for inclusion: cross-sector policy synergies and inter-professional collaboration in and around schools. Bruselas : European Union, DG Education and Culture. Versión electrónica disponible en http://ec.europa.eu/education/library/reports/neset_en.pdf (última consulta 12 mayo 2014)

EURYDICE. (2009). Integrating immigrant children into schools in Europe. Bruselas : European Commission. Versión electrónica disponible en http://eacea.ec.europa.eu/education/eurydice/documents/thematic_reports/101EN.p df (última consulta 12 mayo 2014)

Fullan, M. (2011). Choosing the wrong drivers for whole system reform. Centre for Strategic Education. Seminar series, $\mathrm{n}^{\circ} 204$.

Giménez, C. (2010). El interculturalismo: propuesta conceptual y aplicaciones prácticas. Zarautz : Ikuspegi - Observatorio Vasco de Inmigración.

Hargreaves, A. (2003). Enseñar en la sociedad del conocimiento. Barcelona : Octaedro.

http://www.boe.es/boe/dias/2006/05/04/pdfs/A17158-17207.pdf

http://www.boe.es/boe/dias/2013/12/10/pdfs/BOE-A-2013-12886.pdf

http://www.mecd.gob.es/servicios-al-ciudadano-mecd/dms/mecd/servicios-al-ciudadanomecd/estadisticas/educacion/indicadores-publicaciones-sintesis/datos-cifras/Datosy-Cifras-2013-2014-LR/Datos\%20y\%20Cifras\%202013-2014_final.pdf

Ley Orgánica 2/2006, de 3 de mayo, de educación.

Ley Orgánica 2/2009, de 11 de diciembre, de reforma de la Ley Orgánica 4/2000, de 11 de enero, sobre derechos y libertades de los extranjeros en España y su integración social. http://www.boe.es/boe/dias/2009/12/12/pdfs/BOE-A-2009-19949.pdf

Ley Orgánica 8/2013, de 9 de diciembre, para la mejora de la calidad educativa.

Lyche, C.S. (2010). Taking on the completion challenge. A literature review on policies to prevent dropout and early school leaving. OECD Education working papers, $\mathrm{N}^{\circ} 53$.

MECD. (2013). Datos y cifras. Curso 2013/2014.

Mourshed, M, Chijioke, C. \& Barber, M. (2011). How the world's most improved school systems keep getting better. McKinsey Report, McKinsey Company.

OCDE. (2006). Where immigrant students succeed. Paris : OCDE Publishers. Versión electrónica disponible en http://www.oecd-ilibrary.org/education/where-immigrantstudents-succeed_9789264023611-en;jsessionid=4Inr4pc7w4fi.x-oecd-live-02 (última consulta 12 mayo 2014)

OCDE. (2012). Untapped skills. Realising the potential of immigrant students. Paris : OCDE Publishers. Versión electrónica disponible en 
http://www.oecd.org/education/school/Untapped\%20Skills.pdf (última consulta 12 mayo 2014)

Portes, A. y Aparicio, R. (2013). Investigación longitudinal sobre la segunda generación en España. Avance de resultados. Fundación Ortega-Marañón. Versión electrónica disponible en http://www.ortegaygasset.edu/descargas/pdf_noticias/Resultados\%20ILSEG20130k. pdf (última consulta 12 mayo 2014)

Sánchez Martí, A. y Sandín, M.P. (2013). Joves immigrants i persistència acadèmica: què ens diuen les seves xarxes personals. Temps d'Educació, 44, pp.177-190.

Torrabadella, L. y Tejero, E. (2005). Pioneres i pioners. Trajectòries biogràfiques de filles i fills de famílies immigrades a Catalunya. Barcelona: Fundació caixa Sabadell y Fundació Jaume Bofill. Versión electrónica disponible en http://www.fbofill.cat/intra/fbofill/documents/publicacions/399.pdf (última consulta 12 mayo 2014)

UNESCO. (2008). Inclusive education: the way of the future. Conclusions and recommendations. $48^{\text {th }}$. International Conference on Education. Geneva, 25-28 November 2008. 


\section{Autores}

\section{Miquel Ángel Essomba}

Doctor en pedagogía por la UAB, máster en psicología de la educación y postgraduado en pedagogía intercultural. Actualmente ejerce de profesor agregado (titular) en la Universidad Autónoma de Barcelona, donde dirige el grupo de investigación ERDISC y una cátedra de educación comunitaria. Es miembro del grupo de investigación consolidado EDO - UAB. En el ámbito internacional es coordinador general de la red SIRIUS de políticas sobre migración y educación de la UE. Entre sus últimas publicaciones destacan el libro Inmigración e interculturalidad en la ciudad. Principios, ámbitos y condiciones para una acción comunitaria intercultural en perspectiva europea. (Barcelona, 2012 : Graó) e Inmigración, sociedad y educación en la UE. Hacia una política educativa de plena inclusión, publicado en Cultura y Educación (Vol. 24, n² 2, 2012) 